\title{
TRANSFORMATION OF THE SECOND ORDER BOUNDARY VALUE PROBLEM INTO INTEGRAL FORM - DIFFERENT APPROACHES AND A NUMERICAL SOLUTION
}

\author{
Jaroslaw Siedlecki ${ }^{1}$, Mariusz Ciesielski ${ }^{2}$, Tomasz Btaszczyk ${ }^{1}$ \\ ${ }^{I}$ Institute of Mathematics, Czestochowa University of Technology \\ Częstochowa, Poland \\ ${ }^{2}$ Institute of Computer and Information Sciences \\ Czestochowa University of Technology, Czestochowa, Poland \\ jaroslaw.siedlecki@im.pcz.pl,mariusz.ciesielski@icis.pcz.pl,tomasz.blaszczyk@im.pcz.pl
}

\begin{abstract}
In this paper we present different approaches to the transformation of the second order ordinary differential equation, with respect to adequate boundary conditions, into integral equations. The obtained equations are Fredholm integral equations of the second kind. Next, a numerical method based on quadrature methods has been proposed to get an approximate solution of these equations.
\end{abstract}

Keywords: BVP problem, Fredholm integral equation, Fubini's theorem, numerical methods

\section{Introduction}

In this paper we consider the following ordinary differential equation with adequate boundary conditions on the interval $t \in[a, b]$

$$
D^{2} x(t)+\omega^{2} x(t)=f(t)
$$

where $D^{2} \equiv d^{2} / d t^{2}, x \in C^{2}([a, b])$, and $\omega$ is a constant. The boundary value problem (BVP) (1) is well known and its exact solution may be found in many standard introductions to ordinary differential equations [1-3].

Changing the ordinary differential equations of second order into an integral equation is another possibility of solving the BVP. In several investigations, the second order differential equation (1) is transformed into a Volterra nonhomogeneous integral equation $[4,5]$. This approach is one of the most known but not the only.

In this paper we present different approaches to reduction of the BVP for the second order ordinary differential equation (1) to an integral equation (Volterra or Fredholm). 


\section{Main results}

Let us start from the definition of integral operators (assuming that $I_{a^{+}} x(t)=\int_{a}^{t} x(s) d s$ and $\left.I_{b^{-}} x(t)=\int_{t}^{b} x(s) d s\right)$

$$
\begin{aligned}
& \mathcal{I}_{a^{+}, a^{+}} x(t):=I_{a^{+}} I_{a^{+}} x(t)=\int_{a}^{t} \int_{a}^{u} x(s) d s d u \\
& \mathcal{I}_{a^{+}, b^{x}} x(t):=I_{a^{+}} I_{b^{-}} x(t)=\int_{a}^{b} \int_{u}^{b} x(s) d s d u \\
& \mathcal{I}_{b^{-}, a^{+}} x(t):=I_{b^{-}} I_{a^{+}} x(t)=\int_{t}^{b} \int_{a}^{u} x(s) d s d u \\
& \mathcal{I}_{b^{-}, b^{x}} x(t):=I_{b^{-}} I_{b^{-}} x(t)=\int_{t}^{b} \int_{u}^{b} x(s) d s d u
\end{aligned}
$$

In the following part of the paper we will use the composition rules of operators (2)-(5) with the second order differential operator $D^{2}$

$$
\begin{aligned}
& \mathcal{I}_{a^{+}, a^{+}} D^{2} x(t)=x(t)-x(a)-(t-a) x^{\prime}(a) \\
& \mathcal{I}_{a^{+}, b^{-}} D^{2} x(t)=-x(t)+x(a)+(t-a) x^{\prime}(b) \\
& \mathcal{I}_{b^{-}, a^{+}} D^{2} x(t)=-x(t)+x(b)-(b-t) x^{\prime}(a) \\
& \mathcal{I}_{b^{-}, b^{-}} D^{2} x(t)=x(t)-x(b)+(b-t) x^{\prime}(b)
\end{aligned}
$$

Analysing the above results, we can see that in each case a set of four boundary conditions $\left\{\left\{x(a), x^{\prime}(a)\right\},\left\{x(a), x^{\prime}(b)\right\},\left\{x^{\prime}(a), x(b)\right\},\left\{x(b), x^{\prime}(b)\right\}\right\}$ arises.

Now, we will consider four cases of acting of each integral operator on the considered differential equation (1).

Case 1: By using the integral operator (2) acting on Eq. (1) we obtain

$$
\mathcal{I}_{a^{+}, a^{+}} D^{2} x(t)+\omega^{2} \mathcal{I}_{a^{+}, a^{+}} x(t)=\mathcal{I}_{a^{+}, a^{+}} f(t)
$$

Next, we use the composition rule of operators (6) and finally we get

$$
x(t)+\omega^{2} \mathcal{I}_{a^{+}, a^{+}} x(t)=g_{1}(t)
$$

where $g_{1}(t)=x(a)+(t-a) x^{\prime}(a)+\mathcal{I}_{a^{+}, a^{+}} f(t)$. Eq. (11) is the integral form of the considered equation (1). 
In a similar way, integral operators (3)-(5) acting on Eq. (1) we obtain the following integral equations - cases 2-4.

Case 2: by using operator (3)

$$
x(t)-\omega^{2} \mathcal{I}_{a^{+}, b^{-}} x(t)=g_{2}(t)
$$

where $g_{2}(t)=x(a)+(t-a) x^{\prime}(b)-\mathcal{I}_{a^{+}, b^{-}} f(t)$.

Case 3: by using operator (4)

$$
x(t)-\omega^{2} \mathcal{I}_{b^{-}, a^{+}} x(t)=g_{3}(t)
$$

where $g_{3}(t)=x(b)-(b-t) x^{\prime}(a)-\mathcal{I}_{b^{-}, a^{+}} f(t)$.

Case 4: by using operator (5)

$$
x(t)+\omega^{2} \mathcal{I}_{b^{-}, b^{-}} x(t)=g_{4}(t)
$$

where $g_{4}(t)=x(b)-(b-t) x^{\prime}(b)+\mathcal{I}_{b^{-}, b^{-}} f(t)$.

It is worthwhile to note that Eqs. (11)-(14) are non-homogeneous Fredholm integral equations. One can note that in the all cases one boundary condition of the first kind and one of the second kind occur. If we would like to consider Eq. (1) with boundary conditions only of the first kind on the both boundaries we need tomake simple transformations of one of above integral equations. The aim of these transformations is to determine an unknown value of boundary condition on the basis of the given boundary condition of the first kind.

Using Fubini's theorem $[4,5]$ the integral operators (2)-(5) can be written in the following forms

$$
\begin{aligned}
& \mathcal{I}_{a^{+}, a^{+}} x(t)=\int_{a}^{b} K_{1}(t, s) x(s) d s, \text { with } K_{1}(t, s)=\left\{\begin{array}{cc}
t-s, & \text { if } s \leq t \\
0, & \text { if } s>t
\end{array}\right. \\
& \mathcal{I}_{a^{+}, b^{-}} x(t)=\int_{a}^{b} K_{2}(t, s) x(s) d s, \text { with } K_{2}(t, s)= \begin{cases}s-a, & \text { if } s \leq t \\
t-a, & \text { if } s>t\end{cases} \\
& \mathcal{I}_{b^{-}, a^{+}} x(t)=\int_{a}^{b} K_{3}(t, s) x(s) d s, \text { with } K_{3}(t, s)= \begin{cases}b-t, & \text { if } s \leq t \\
b-s, & \text { if } s>t\end{cases} \\
& \mathcal{I}_{b^{-}, b^{-}} x(t)=\int_{a}^{b} K_{4}(t, s) x(s) d s, \text { with } K_{4}(t, s)=\left\{\begin{array}{cc}
0, & \text { if } s \leq t \\
s-t, & \text { if } s>t
\end{array}\right.
\end{aligned}
$$

It should be noted that the following properties occur 


$$
\begin{aligned}
& \mathcal{I}_{a^{+}, a^{+}} x(t)+\mathcal{I}_{b^{-}, a^{+}} x(t)=\int_{a}^{b}(b-s) x(s) d s=C \text { for arbitrary } a \leq t \leq b \\
& \mathcal{I}_{a^{+}, b^{x}} x(t)+\mathcal{I}_{b^{-}, b^{-}} x(t)=\int_{a}^{b}(s-a) x(s) d s=C \text { for arbitrary } a \leq t \leq b
\end{aligned}
$$

Let us write the above integral equations (11)-(14) in a more general form

$$
x(t) \pm \int_{a}^{b} K_{m}(t, s) x(s) d s=g_{m}(t), \quad \text { for } m=1, \ldots, 4
$$

where $g_{m}(t)$ are the right-hand sides of equations (11)-(14), respectively.

\section{Numerical solution}

We solve Eqs. (11)-(14) by using the Nystrom method [6, 7]. The integral operators (15)-(18) we approximate using a quadrature rule

$$
\int_{a}^{b} K_{m}(t, s) x(s) d s \cong \sum_{j=0}^{n} K_{m}\left(t, s_{j}\right) x\left(s_{j}\right) w_{j}
$$

where $w_{j}, j=0,1, \ldots, n$ are the weights of the quadrature rule and $n+1$ is a number of discretised points. We solve Eq. (21) on a mesh with uniform spacing: $t_{i}=a+i \Delta t, i=0,1, \ldots, n$, and $\Delta t=(b-a) / n$. We can choose one of many known quadratures. For example, for the trapezoidal method we assume: $w_{0}=w_{n}=\Delta t / 2$ and $w_{j}=\Delta t$, for $j=1, \ldots, n-1$.

We evaluate equations (21) for $m=1, \ldots, 4$ at the quadrature points in the form

$$
x\left(t_{i}\right) \pm \omega^{2} \sum_{j=0}^{n} K_{m}\left(t_{i}, s_{j}\right) x\left(s_{j}\right) w_{j}=g_{m}\left(t_{i}\right)
$$

In every function $g_{m}$ the part with integral operator of function $f$ occurs. If $f$ is a simple analytical function then one can try to find the analytical formula. In the general case, the numerical formula (22) is useful.

In matrix notation, the equations (23) can be written as

$$
\left(\mathbf{I} \pm \omega^{2} \overline{\mathbf{K}}_{m}\right) \cdot \mathbf{x}=\mathbf{g}_{m}
$$

where $\mathbf{x} \quad$ and $\quad \mathbf{g}_{m} \quad$ are the vectors $\quad \mathbf{x}=\left[x\left(t_{0}\right), \ldots, x\left(t_{i}\right), \ldots, x\left(t_{n}\right)\right]^{T}$, $\mathbf{g}_{m}=\left[g_{m}\left(t_{0}\right), \ldots, g_{m}\left(t_{i}\right), \ldots, g_{m}\left(t_{n}\right)\right]^{T}$ and $\overline{\mathbf{K}}_{m}$ is matrix $\overline{\mathbf{K}}_{m}=\left\{\left(K_{m}\right)_{i, j} \cdot w_{j}\right\}_{(n+1) \times(n+1)}$ 
and $\left(K_{m}\right)_{i, j} \equiv K_{m}\left(t_{i}, s_{j}\right)$ for $i=0, \ldots, n$ and $j=0, \ldots, n$. In this way we obtain a system of $n+1$ linear algebraic equations in $n+1$ unknowns.

\section{Example of numerical simulations}

For implementing the presented method for solving Fredholm integral equations for four cases, we choose the example for which the exact solution is known (for comparison with the approximate solutions). Let us assume that the analytical solution of Eq. (1) for $\omega=\pi$ and $f(t)=2 \pi \sin (\pi t)$ is of the form

$$
x(t)=-t \cos (\pi t)
$$

and on this basis, we determine a set of the boundary conditions for $a=1$ and $b=3: x(a)=1, x(b)=3, x^{\prime}(a)=1$ and $x^{\prime}(b)=1$.

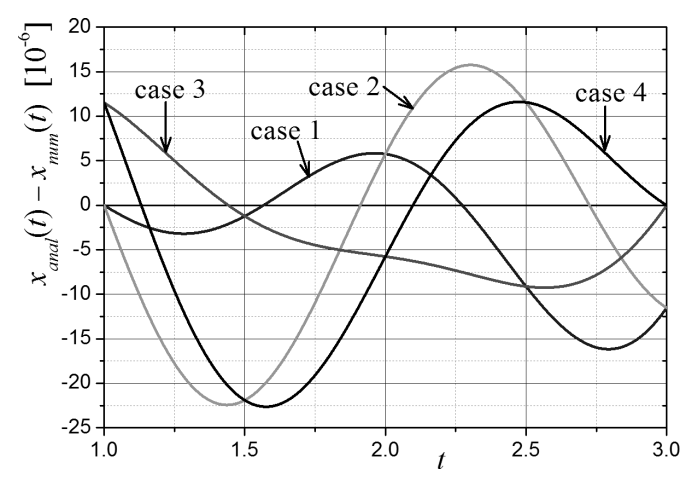

Fig. 1. Plots of differences between analytical solution (25) and four cases of numerical solutions of Eq. (1) for $n=1000$

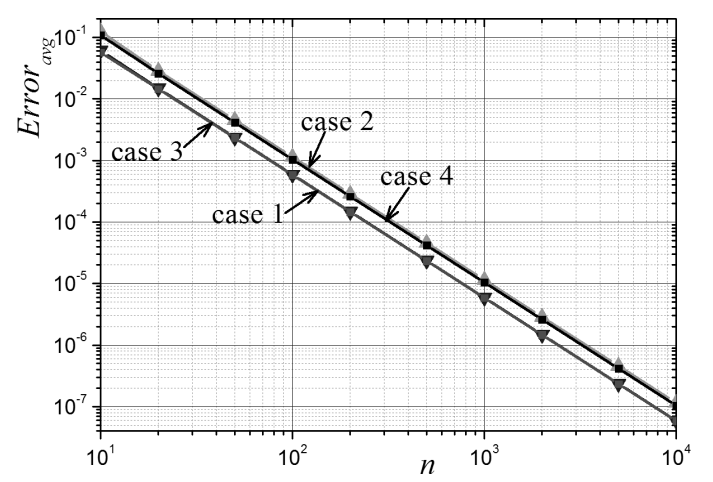

Fig. 2. Average errors of numerical solutions for different values of $n$ 
The numerical solutions of integral equations (11)-(14) have been evaluated for four cases. In the all cases, these solutions should be close to the given analytical solution (25). In Figure 1 we show the computed errors as differences between analytical and numerical solutions: $x_{\text {nnal }}\left(t_{i}\right)-x_{\text {num }}\left(t_{i}\right)$ obtained for $n=1000$. The Figure 2 presents the values of average errors defined as

$$
\operatorname{Error}_{\text {avg }}(n)=\frac{1}{n+1} \sum_{i=0}^{n}\left|x_{\text {anal }}\left(t_{i}\right)-x_{\text {num }}\left(t_{i}\right)\right|
$$

for different values of parameter $n$. One can see that the behaviour of the numerical solutions as we increase $n$ gives smaller errors (as expected).

\section{Conclusions}

In this work, we applied various ways of integration of the second order nonhomogenous differential equation. In this way we obtained four cases of Fredholm integral equations of the second kind with different kernels. Each case concerns the application of a set of adequate boundary conditions. The advantage of this approach is boundary conditions applied in a straightforward manner which will be satisfied by the considered BVP. Next, numerical method based on quadrature methods has been proposed to approximate the solution of these Fredholm integral equations. From the results of numerical examples, one can note that the proposed numerical method is efficient and accurate to estimate the solution of these integral equations. Moreover, we show that when the value of mesh step decreases, the errors decrease to the small values.

\section{References}

[1] Agarwal R.P., O'Regan D., An Introduction to Ordinary Differential Equations, Springer, New York 2008.

[2] Kukla S., Funkcje Greena i ich zastosowania, Wyd. Politechniki Częstochowskiej, Częstochowa 2009.

[3] Zaitsev V.F., Polyanin A.D., Handbook of Exact Solutions for Ordinary Differential Equations, CRC Press, New York 1995.

[4] Collins P.J., Differential and Integral Equations, Oxford University Press, New York 2006.

[5] Polyanin A.D., Manzhirov A.V., Handbook of Integral Equations (Second Edition), Taylor \& Francis Group, Boca Raton 2008.

[6] Press W.H., Teukolsky S.A., Vetterling W.T., Flannery B.P., Numerical Recipes: The Art of Scientific Computing (3rd ed.), Cambridge University Press, New York 2007.

[7] Atkinson K.E., The Numerical Solution of Integral Equations of the Second Kind, Cambridge University Press, 2009. 